\section{Retinal nerve fibre layer loss in diabetes mellitus without retinopathy}

\section{B Skarf}

New study provides no new information

M any diabetics worldwide might benefit if a simple, reliable, objective, and non-invasive technical method were available to detect and monitor the development of retinopathy and visual deficits without requiring a professional examination. With this ultimate goal in mind, Lopes de Faria and colleagues describe their initial use of scanning laser polarimetry (SLP) to measure retinal nerve fibre layer thickness in diabetics without retinopathy in this issue of the BJO (p 725).

Evaluation of the retinal nerve fibre layer (RNFL) as a means of assessing optic nerve health has been a clinical and investigational tool for at least 30 years. Ophthalmoscopic scrutiny of the nerve fibre layer was first advocated by Hoyt $\mathrm{t}^{1-3}$ and this approach was soon enhanced by the description of photographic manipulations designed to increase the contrast between retinal areas with different nerve fibre layer thicknesses. ${ }^{4}$ For most ophthalmologists, these qualitative methods were difficult to apply and had limited usefulness in monitoring progression of disease in a clinical setting. Furthermore, they depended greatly on the clarity of the ocular media. Now, with instruments capable of objective determination of nerve fibre layer thickness, substantial interest has focused on the significance of early thinning of the nerve fibre layer in glaucoma, papilloedema, and a variety of other optic neuropathies.

Lopes de Faria and co-workers used the GDx nerve fibre analyser (Laser Diagnostic Technologies, San Diego, USA) to provide the first quantitative assessment of nerve fibre layer thickness in diabetics. Their small sample of 12 patients, between the ages of 18 and 40 , have had type 1 diabetes mellitus for a minimum of 10 years, with no retinopathy detectable by clinical examination or fluorescein angiography (level 1, modified Airlie House classification). In comparing this sample with an age and sex matched control group without diabetes, the authors demonstrated that the diabetic group has statistically significant thinning of the nerve fibre layer in the quadrant superior to the optic disc. There is no statistical difference between control and diabetic group data for nasal, temporal, or inferior retinal quadrants. The authors offer no explanation for this selective topographic difference in RNFL thinning.

\section{The usefulness of SLP in the early detection of RNFL thinning in diabetes should not be inflated}

Although interesting because of the use of an objective technique, this study provides no new information. It confirms what we have known for 8 years from the only other published studies that describe RNFL findings in diabetics without retinopathy ${ }^{56}$ (appropriately cited by our authors).

The significance of this finding and the usefulness of SLP in the early detection of RNFL thinning in diabetes should not be inflated. Firstly, the sample chosen for this study is very small and though the statistical analysis shows significance at the $\mathrm{p}=0.03$ level, the statistical approach used in this study is suboptimal. Given the multiple comparisons that were made between different sets of data, the probability that one of the measures would have been falsely positive was increased by the approach used. A multivariate analysis would have been more appropriate. It is also worth noting that for two pairs of data sets (inferior and nasal polar integrals) the $\mathrm{SD}$ of the data for one group (that is, patient or control) is an order of magnitude larger than the SD of the data from the other group. Thus, data for inferior and nasal polar integral measures could not possibly achieve statistical significance. Furthermore, review of the data presented in Figure 2 shows that all except two or three of the patients studied fall within 2.5 SD of the mean of the control group. Thus, these data will not allow the authors (or us) to determine whether each individual specific measure of superior RNFL thickness in most of their diabetic patients is abnormal.

In conclusion, this study, supporting earlier qualitative observation, hints at the possibility that careful RNFL thickness measurements might help detect early changes in diabetics. We are still no closer to recognising these measures as being clinically useful and it would be premature to begin evaluating the RNFL of individual diabetic patients using these techniques. Properly designed, prospective, longitudinal clinical studies on larger populations of patients without retinopathy are mandated before we can accept and recommend the diagnostic and predictive value of RNFL measures made by this or any other methodology.

Br J Ophthalmol 2002;86:709

\section{Author's affiliations}

B Skarf, Department of Ophthalmology, Henry Ford Health Sciences Center, Detroit, Michigan, USA; bskarf1@hfhs.org

\section{REFERENCES}

1 Hoyt WF, Schlicke B, Eckelhoff RJ. Fundoscopic appearance of a nerve-fibre-bundle defect. Br J Ophthalmol 1972;56:577-83.

2 Hoyt WF, Frisén L, Newman NM. Funduscopy of nerve fiber layer defects in glaucoma. Invest Ophthalmol 1973;12:814-29.

3 Hoyt WF. Fundoscopic changes in the retinal nerve-fibre layer in chronic and acute optic neuropathies. Trans Ophthalmol Soc UK 1976;96:368-71.

4 Miller NR, George TW. Monochromatic photography and ophthalmoscopy of the peripapillary retinal nerve fiber layer. Invest Ophthalmol 1997;17:1 121-3

5 Chihara E, Matsuoka T, Ogura Y, et al. Retinal nerve fiber layer defect as an early manifestation of diabetic retinopathy. Ophthalmology 1993;100:1147-51.

6 Bartz-Schmidt KU, Schmitz-Valckenberg P. Retinal nerve fiber layer photography and papillometry in juvenile diabetes mellitus. Ophthalmology 1994;91:364-7. 


\section{Surgery for glaucoma in the $21 \mathrm{st}$ century}

\section{P T Khaw, A P Wells, K S Lim}

\section{How close are we to a utopian world?}

M

any of us who were brought up in the latter half of the 20th century will recall reading and watching science fiction programmes in the media predicting life in the 21 st century. Apart from a life of leisure facilitated by intelligent computers and robots, we were meant to enjoy a prolonged life because of advances in science and technology. The few of us who did not have our genetic defects and predisposition to various diseases ironed out by the new "molecular surgery" would enjoy surgical correction with advanced technology including magic scalpels which left no scar. The surgery itself would be quick, have minimal complications, and require no postoperative care and manipulation, achieving the desired surgical "end point" in 95-100\% of cases. Cataract surgery is nearly there, so how close are we to this utopian world with regard to glaucoma surgery?

In this issue of the BJO (p 748) O'Brart and colleagues report their randomised prospective study comparing viscocanalostomy with antimetabolite assisted trabeculectomy. In theory, the operation of viscocanalostomy is claimed to bring us one step closer to the restoration of normal drainage by dilating (with viscoelastic) and facilitating flow round Schlemm's canal without macroscopically penetrating the eye. ${ }^{1}$ There is some evidence that the dilatation of Schlemm's canal can be achieved at least for a short period with a viscoelastic injection, although the value of this is uncertain.

However, deroofing of Schlemm's canal and creation of a scleral lake are also carried out at the same time and there is anecdotal evidence that viscocanalostomy does not work without this component of the surgery. This component on its own is the operation known as non-penetrating deep sclerectomy. In theory, these procedures drain aqueous into scleral veins within the scleral lake rather that subconjunctivally in conventional drainage surgery, and it has been postulated that this may reduce the need for antiscarring agents. Leaving a remnant of meshwork may allow resistance to outflow, therefore reducing hypotony, preserving the blood-aqueous barrier, and possibly reducing long term cataract. In theory, minimal postoperative follow up is required as scleral flap manipulations used in conventional surgery cannot be done apart from Nd-YAG lasering of the remaining meshwork. So is this the way glaucoma surgery should be going in the 21 st century?

Certainly, viscocanalostomy seems to have advantages compared to trabeculectomy in the early postoperative period with fewer complications such as transient hyphaema, anterior chamber shallowing and bleb leak. However, these were all transient. What is more relevant is that there were five postoperative cataracts requiring surgery in the trabeculectomy group $(60 \%$ of whom had shallow anterior chambers and lower intraocular pressures in the early postoperative period) although intraocular pressure control was maintained after cataract surgery.

\section{We have yet to achieve ideal glaucoma surgery}

However, there were disadvantages to viscocanalostomy, which means it does not achieve the desired criteria for $21 \mathrm{st}$ century surgery at present. At 12 months $26 \%$ of the viscocanalostomy eyes had intraocular pressures of $<15 \mathrm{~mm} \mathrm{Hg}$ versus $76 \%$ of the trabeculectomy group, although postoperative Nd-YAG goniotomy was not performed in the first year, which has improved the success rate in other series of non-penetrating surgeries. This level of intraocular pressure control is now known to be associated with minimal glaucoma progression over nearly a decade of follow up. ${ }^{2}$ This finding of a higher "failure" rate based on intraocular pressure after "nonpenetrating" surgery compared with trabeculectomy has been a finding in the majority of randomised trials comparing the two procedures. ${ }^{3-5}$

Viscocanalostomy or other similar procedures are not at present the procedure to replace trabeculectomy. Yet comparative studies offer us insights as to how we can move closer to this ideal. The early hypotony seen after trabeculectomy appears to play a significant part in cataract formation. This hypotony leads to a breakdown of the blood-aqueous barrier (albeit for a short time) which leads to leakage of growth factors, of which transforming growth factor $\beta$ (TGF- $\beta$ ) is known to be particularly cataractogenic. ${ }^{6}$ This growth factor is autoinductive so even a short exposure can set off a self amplifying and perpetuating chain of events leading to cataract. It may be possible to neutralise this and other cataractogenic molecules with antibodies. $^{7}$

Using relatively simple measures, it is now possible to prevent early postoperative hypotony after trabeculectomy in the perioperative and early postoperative period using a simple intraoperative anterior chamber infusion and releasable/adjustable sutures. The advantage of adjustable sutures is the pressure can be more easily adjusted down to the desired level postoperatively. It will be interesting to look at the incidence of cataract in patients in whom the early intraocular pressure has been very tightly controlled after trabeculectomy.

The presence of a bleb in patients undergoing viscocanalostomy strongly suggests that subconjunctival drainage is occurring, which is likely given that it is almost impossible to secure the outer scleral flap in non-penetrating surgery after a large inner scleral block has been removed. Therefore, accidental macroperforation (as opposed to microperforation which occurs during the deroofing procedure) of the meshwork leads to a high incidence of early hypotony. The more desirable diffuse blebs seen with viscocanalostomy can now be achieved with trabeculectomy, even with adjunctive mitomycin $\mathrm{C}$, by using a larger scleral flap and a larger area of mitomycin C treatment, which considerably reduces bleb related complications. ${ }^{8}$ This also suggests that scarring processes may be important in non-penetrating techniques, and that the use of antiscarring techniques may increase the success rate of this surgery. Great care has to be taken if antimetabolites are used in nonpenetrating surgery because of the danger of intraoperative intraocular penetration given the very deep dissections. Newer, less toxic antiscarring agents may improve this situation in the future. $^{7}$

What is clear is that we are still some way from the perfection expected from surgery in the 21 st century. However, simple methods are already available that could considerably improve the way we carry out trabeculectomy, which at presents appears much more likely to achieve the pressure reductions associated with arrest of glaucoma. We should, however, continue to strive to improve glaucoma surgery until we come close to 
the perfection we so desire. Current tube drainage devices do not meet this expectation ${ }^{9}$ and there are considerable technical obstacles to be overcome until we can ensure that they do. ${ }^{10}$

The keys to this ideal surgery lie in our ability to understand and control two processes-aqueous flow and the healing response of the eye. We must therefore be open to advances in all fields including less popular areas such as aqueous flow regulation, and newer areas including innovative surgical techniques, biological materials, nanotechnology, and molecular and cell biology. Only then will we be carrying out a 5 minute "procedure" with $95+\%$ of patients achieving pressures in the 10-12 $\mathrm{mm} \mathrm{Hg}$ range for several decades after surgery without complications (while the nerve is being regenerated). Impossible? Only if we think so.

\section{ACKNOWLEDGEMENTS}

The authors are supported by the Medical Research Council (UK) grant G9330070, Department of Health/Department of Trade and Industry link award, and the International Glaucoma Association.

Br J Ophthalmol 2002;86:710-711

..................

Authors' affiliations

P T Khaw A P Wells, K S Lim, Glaucoma and Wound Healing Research Units, MRC 5-FU Clinical Trial Team, Moorfields Eye Hospital and the Institute of Ophthalmology, University College London, UK

Correspondence to: Professor P T Khaw, Wound Healing Research Unit, Glaucoma Unit and Divisions of Pathology and Cell Biology, Moorfields Eye Hospital and Institute of Ophthalmology, Bath Street London ECIV 9EL, UK; p.khaw@ucl.ac.uk

\section{REFERENCES}

1 Stegmann R, Pienaar A, Miller D. Viscocanulostomy for open angle glaucoma in black African patients. J Cataract Refract Surg 1999;25:316-22.

2 AGIS Investigators. The Advanced Glaucoma Intervention study (AGIS) 7. The relationship between control of intraocular pressure and visual field deterioration. Am J Ophthalmol 2000;130:429-40

3 Chiselita D. Non-penetrating deep sclerectomy versus trabeculectomy in primary open angle glaucoma surgery. Eye 2001:15:197-201.

4 El Sayyad F, Helal M, El Kholify $\mathrm{H}$, et al. Nonpenetrating deep sclerectomy versus trabeculectomy in bilateral primary open angle glaucoma. Ophthalmology 2000; 107:1671-4.

5 Jonescu-Cuypers C, Jacobi PC, Konen W, et al. Primary viscocanalostomy versus trabeculectomy in white patients with open-angle glaucoma. A randomised clinical trial. Ophthalmology 2001;108:254-8.

6 Hales AM, Chamberlain CG, McAvoy JW. Cataract induction in lenses cultures with transforming growth factor-beta. Invest Ophthalmol Vis Sci 1995:36:1709-13.

7 Siriwardena D, Khaw PT, King AJ, et al. A Siriwardena D, Khaw $\mathrm{PT}$, King $A$, et al. A
randomised double-masked placebo-contolled trial of human anti-TGFbeta2 monoclonal antibody (CAT-152): a potential new modulator of wound healing following trabeculectomy. Ophthalmology 2002;109:427-31.

8 Wells AP, Cordeiro MF, Bunce CV, et al. Cystic bleb related complications in limbus versus fornix based flaps in paediatric and young adult trabeculectomy with high dose mitomycin C. Invest Ophthalmol Vis Sci 2001;42:S544.

9 Lim KS, Allan BDS, Lloyd AW, et al. Glaucoma drainage devices; past, present and future. $\mathrm{Br} J$ Ophthalmol 1998;92:1083-9.

10 The AGFID project team. Experimental flow studies in glaucoma drainage device development. Br J Ophthalmol 2001;85:1231-6.

\section{Clinical Evidence-Call for contributors}

Clinical Evidence is a regularly updated evidence based journal available world wide both as a paper version and on the internet. Clinical Evidence urgently needs to recruit a number of new contributors. Contributors are health care professionals or epidemiologists with experience in evidence based medicine and the ability to write in a concise and structured way.

We are presently interested in finding contributors with an interest in the following clinical areas:

$\begin{array}{ll}\text { Acute bronchitis } & \text { Hepatitis B } \\ \text { Acute sinusitis } & \text { Hepatitis C } \\ \text { Cataract } & \text { HIV } \\ \text { Genital warts } & \end{array}$

Being a contributor involves:

- Appraising the results of literature searches (performed by our Information Specialists) to identify high quality evidence for inclusion in the journal.

- Writing to a highly structured template (about 1500-3000 words), using evidence from selected studies, within 6-8 weeks of receiving the literature search results.

- Working with Clinical Evidence Editors to ensure that the text meets rigorous epidemiological and style standards.

- Updating the text every eight months to incorporate new evidence.

- Expanding the topic to include new questions once every 12-18 months.

If you would like to become a contributor for Clinical Evidence or require more information about what this involves please send your contact details and a copy of your CV, clearly stating the clinical area you are interested in, to Polly Brown (pbrown@bmigroup.com). 